\title{
Public Health Ausbildung in Österreich. Ein Überblick
}

\author{
Günter Diem · Thomas Ernst Dorner
}

Eingegangen: 7. Oktober 2013 / Angenommen: 26. März 2014 / Online publiziert: 9. April 2014

(C) Die Autor(en) 2014. Dieser Artikel ist auf Springerlink.com mit Open Access verfügbar.

Zusammenfassung Die Herausforderungen, die auf das österreichische Gesundheitssystem zukommen, benötigen eine steigende Zahl an Public Health Expertinnen und Experten aus vielen Professionen und in allen politischen und gesellschaftlichen Sektoren. Dieser Artikel befasst sich mit der Recherche über das Angebot an Public Health Ausbildungen an österreichischen Universitäten und Fachhochschulen anhand der vorwiegend online vorhandenen Informationen auf den Web-Plattformen der Hochschuleinrichtungen. In Österreich gibt es derzeit (Stand 2013) drei postgraduelle Public Health Universitätslehrgänge und zwei Public Health Doktoratsstudien. Weiters konnten 34 Studiengänge identifiziert werden, in denen Teilbereiche von Public Health abgedeckt sind. Public Health Inhalte haben auch in die Ärzteausbildungscurricula an den österreichischen medizinischen Universitäten Einzug gefunden. In Österreich gibt es bereits ein vielfältiges Angebot für Public Health Aus- und Weiterbildung. Um eine adäquate Public Health Workforce zu bilden, die im Stande ist die Public Health Herausforderungen in allen Dimensionen im Sinne von Health in All Policies zu meistern, sollte dieses Angebot jedoch noch intensiviert werden.

Schlüsselwörter Public Health · Ausbildung · Universität $\cdot$ Fachhochschule $\cdot$ Lehrgang

Dr. med. G. Diem, MD, MPH $(\bowtie) \cdot$ T. E. Dorner

Austrian Public Health Society,

Millöckergasse 19,

6850 Dornbirn, Österreich

E-Mail: guenter.diem@diemphd.com

\section{T. E. Dorner}

Institute of Social Medicine, Centre for Public Health, Medical University Vienna,

Wien, Österreich

\section{Public Health Education in Austria. An overview}

Summary The future challenges for the Austrian health care system require an increasing number of public health experts of different professions in all fields of public health. In this article the offer of public health education in Austrian universities and universities for applied sciences was searched based on the predominantly online available information on web platforms of the schools. Currently (2013), there are three postgraduate public health university courses and two public health doctoral programs in Austria. Additionally, 34 degree programmes could be identified, in which parts of public health are covered. But also in medical curricula at Austrian medical schools, public health contents have found their place. In Austria, there is already a multifaceted offer for public health education. However, to build an appropriate public health work force, capable to manage the public health challenges in all its dimensions in terms of health in all policies, this offer should still be intensified.

Keywords Public health · Education · University · Course

\section{Einleitung}

Die Herausforderungen im Bereich des Gesundheitswesens sind vielfältig, komplex und ändern sich rasch. Weltweit gibt es Stimmen, die nach der Bildung einer Public Health Workforce mit einer adäquaten Ausbildung im Bereich Public Health rufen, um diesen Herausforderungen gerecht zu werden. Für eine Stärkung von Public Health mit dem Ziel, dass Entscheidungen im Gesundheitsbereich wissenschaftlichen Kriterien folgen, braucht es entsprechende interessante Angebote an Public Health Ausbildungsmöglichkeiten. Es sollten 
sowohl neu einsteigende Studierende angesprochen werden als auch Weiterbildungsangebote für bereits im Gesundheitswesen tätige Personen geschaffen werden [1]. Public Health ist ein großes Fachgebiet mit vielen Disziplinen, von Gesundheitsförderung über Gesundheitsökonomie, Versorgungsforschung, Epidemiologie, Gesundheitsmanagement und Gesundheitspolitik bis hin zu Medizin. Für dieses breite Feld braucht es auch vielfältige Ausbildungsangebote.

Public Health Ausbildung und Weiterbildung haben in Österreich noch keine sehr lange Vergangenheit. Der Mangel an personellen Public Health Ressourcen ist seit dem Ende der 1990er Jahre wiederholt artikuliert worden und führte auch zu einer Reihe an Ausbildungsinitiativen seit dem Jahrtausendwechsel. Wissen - als „einzige Ressource, die sich beim Gebrauch vermehrt“ [2]- ist eine unabdingbare Voraussetzung für zielgerichtetes Handeln im Gesundheitssystem, organisatorisch und politisch. Im Bereich Public Health muss daher problemorientiertes, wissenschaftlich fundiertes und sozial verankertes Wissen Gegenstand der Aus- und Weiterbildung sein. Dies stellt zweifellos beträchtliche Anforderungen an die Anbieter von Public Health Ausbildungen dar.

Dieser Artikel gibt einen Überblick über die bestehenden Angebote im Bereich Public Health an österreichischen Universitäten und Fachhochschulen. Er betrachtet die reinen Public Health Lehrgänge näher, beschreibt aber auch, inwieweit Public Health in den bestehenden medizinischen Curricula etabliert ist. In den letzten Jahren haben sich in Österreich einige Ausbildungscurricula im Bereich Public Health gebildet. Lehrgänge und Ausbildungsangebote an Universitäten und Fachhochschulen sind entstanden, die zum Teil Public Health als einzigen Gegenstand, zum Teil Public Health als wichtigen Teil der Ausbildung ins Curriculum integrierten. Zudem wurde auch in den etablierten Medizincurricula die Wichtigkeit von Public Health erkannt und Public Health in die universitäre Ausbildung von Ärztinnen und Ärzten integriert.

Ziel des Artikels ist es also, die bestehenden Kurse für Public Health in Österreich aufzulisten und bei reinen Public Health Kursen deren Inhalt zu beschreiben. Es ist nicht Ziel der Arbeit die Qualität der Lehre und die Relevanz der Inhalte zu bewerten.

\section{Methode}

Anhand verschiedener Online-Plattformen der Lehrgangsanbieter [3, 4] und von Studienratgebern [5] wurde nach Studienangeboten gesucht und die online verfügbaren Informationen über die Studiengänge verglichen. Die Studiengänge wurden auf Lehrinhalte, pädagogische Methoden, Zusammensetzung der Fakultät, Aufnahmekriterien, Zahl der European Credit Transfer System Punkte, Unterrichtsform und andere Merkmale untersucht und miteinander verglichen, sofern diese Informationen verfügbar waren. Das Hochschulangebot in Österreich umfasst 22 Universitäten, 22 Fachhochschulen und 12 Privatuniversitäten (Stand Juli 2013) [6]. Die angebotenen Lehrgänge wurden in erster Linie aufgrund der online verfügbaren (also für zukünftige Studierende zugänglichen) Informationen analysiert und damit bewusst die Perspektive der nach Angeboten suchenden, potentiellen Studierenden eingenommen. Die Beschreibung der Ausbildungsangebote beschränkt sich dementsprechend auf die im Internet bereitgestellte Information.

Ein-/Ausschlusskriterien: In die Liste der reinen Public Health Angebote (Tab. 1) wurden ausschließlich Lehrgänge von Einrichtungen aufgenommen, die in der oben erwähnten Liste des Bundesministeriums für Wissenschaft und Forschung unter „Hochschulen“ aufgelistet werden. Der Lehrgangstitel musste die Bezeichnung „Public Health“ enthalten.

Eine zweite Übersicht (Tab. 2) listet jene Lehrgänge auf, die relevante Teilbereiche der Public Health integrieren und zumindest einen Bachelorabschluss anbieten. Auch hier wurden ausschließlich Angebote aus dem Universitäts- und Hochschulbereich eingeschlossen. Der Lehrgangstitel musste die Bezeichnung „Public Health“, „Health“ oder „Gesundheits-“ enthalten oder das Curriculum eine inhaltliche Annäherung an relevante Public Health Inhalte bieten.

\section{Ergebnisse}

Tabelle 1 gibt einen Überblick über die reinen Public Health Ausbildungen in Österreich. Derzeit werden drei

Tab. 1 Public Health Ausbildungen an Österreichischen Universitäten und Hochschulen

\begin{tabular}{|c|c|c|c|c|}
\hline Veranstalter & Lehrgangsbezeichnung & ECTS & Abschluss & Kosten $(€)^{a}$ \\
\hline \multicolumn{5}{|c|}{ Universitätslehrgänge, postgraduate und berufsbegleitend } \\
\hline Medizinische Universität Wien/Universität Wien & Public Health & 120 & MPH & $13.400,-$ \\
\hline Medizinische Universität Graz & Public Health & 120 & MPH & $12.000,-$ \\
\hline Medizinische Universität Graz/Schloss Hofen Vbg. & Public Health Governance & 90 & MSc PHG & $11.700,-$ \\
\hline \multicolumn{5}{|l|}{ Doktoratsstudien } \\
\hline Medizinische Universität Wien & $\begin{array}{l}\text { Doctoral Program of Applied Medical } \\
\text { Science; Thematic program: Public Health }\end{array}$ & 180 & Dr. scient. med. & - \\
\hline UMIT Tirol & Doktoratsstudium Public Health & 180 & Dr. phil. & $21.600,-$ \\
\hline
\end{tabular}


Tab. 2 Ausbildungen an Österreichischen Universitäten und Hochschulen, die Teilbereich von Public Health abdecken

\begin{tabular}{|c|c|c|c|c|}
\hline Veranstalter & Lehrgangsbezeichnung & ECTS & Abschluss & Kosten $(€)^{c}$ \\
\hline \multicolumn{5}{|c|}{ Universitätslehrgänge, postgraduate und berufsbegleitend } \\
\hline Medizinische Universität Wien & Health Care Management & 90 & MBA & $15.000,-$ \\
\hline Medizinische Universität Wien/Universität Basel & Master of Insurance Medicine ${ }^{\mathrm{a}}$ & 60 & MAS & $27.556,-$ \\
\hline Donauuniversität Krems & Gesundheitsmanagement und Public Health & 60 & $\begin{array}{l}\text { Akademische/r Ex- } \\
\text { pert/e/in in Healthcare } \\
\text { Management }\end{array}$ & $8.500,-$ \\
\hline Donauuniversität Krems & Gesundheitsmanagement und Public Health & 90 & MSc & $11.500,-$ \\
\hline Donauuniversität Krems & Gesundheitsmanagement und Public Health & 120 & MBA & $14.500,-$ \\
\hline Donauuniversität Krems & Management im Gesundheitswesen/Healthcare Management & 120 & MBA & $14.500,-$ \\
\hline Donauuniversität Krems & Gesundheitspädagogik/Health Education & 120 & MSc & $8.500,-$ \\
\hline Medizinische Universität Graz/FH Joanneum & MBA in health care and hospital management & 180 & MBA & $14.500,-$ \\
\hline Medizinische Universität Graz & MSc in Health Education & 120 & MHE & $13.000,-$ \\
\hline Universität Salzburg & MSC Health and Fitness & 90 & $\begin{array}{l}\text { Master of Science } \\
\text { health and Fitness }\end{array}$ & $6.000,-$ \\
\hline Paracelsus Medizinische Privatuniversität & MBA Health Care Management & 90 & MBA & $25.200,-$ \\
\hline Fachhochschule Oberösterreich & Integrated Care Systems (ICS) ${ }^{a}$ & 120 & MAS & $14.160,-$ \\
\hline Pädagogische Hochschule Steiermark & Gesundheitsförderung und Gesundheitspädagogik & 120 & MA & $9.200,-$ \\
\hline \multicolumn{5}{|l|}{ Bachelorstudien } \\
\hline FH Burgenland & Gesundheitsmanagement und Gesundheitsförderung & 180 & BA & - \\
\hline Medizinische Universität Graz & Bachelor Gesundheits- und Pflegewissenschaft & 180 & BSc & - \\
\hline Fachhochschule Kärnten & Bachelorstudium Gesundheits- und Pflegemanagement & 180 & BA & $2.180,16$ \\
\hline IMC Fachhochschule Krems & Gesundheitsmanagement & 180 & BA & $2.180,16$ \\
\hline Fachhochschule Joanneum & Gesundheitsmanagement im Tourismus (Bachelor) & 180 & BA & - \\
\hline MCI Management Center Innsbruck & $\begin{array}{l}\text { Bachelorstudium Nonprofit-, Sozial-, und Gesundheitsma- } \\
\text { nagement }\end{array}$ & 180 & BA & $2.178,-$ \\
\hline $\begin{array}{l}\text { FFH Gesellschaft zur Erhaltung und Durchfüh- } \\
\text { rung von Fachhochschul-Studiengängen mbH }\end{array}$ & Public Health and Aging Management ${ }^{b}$ & 180 & BA & $2.180,16$ \\
\hline \multicolumn{5}{|l|}{ Masterstudien } \\
\hline FH Burgenland & Management im Gesundheitswesen & 120 & MA & - \\
\hline Universität Wien & $\begin{array}{l}\text { Masterstudium Ernährungswissenschaften; Public Health } \\
\text { Nutrition }\end{array}$ & 120 & MSc & - \\
\hline Universität Wien & $\begin{array}{l}\text { European Master in Health and Physical Activity (Joint Curri- } \\
\text { cula); Fitness, Prävention und Public Health }\end{array}$ & 120 & MSc & - \\
\hline Medizinische Universität Graz & Master Gesundheits- und Pflegewissenschaft & 120 & MSc & - \\
\hline Fachhochschule Kärnten & Masterstudiengang Gesundheitsmanagement & 120 & MA & $1.453,44$ \\
\hline Fachhochschule Joanneum & Gesundheitsmanagement im Tourismus (Master) & 120 & MA & - \\
\hline MCI Management Center Innsbruck & Master program International Health and Social Management & 120 & MA & $1.452,-$ \\
\hline $\begin{array}{l}\text { FHG - Zentrum für Gesundheitsberufe Tirol } \\
\text { GmbH }\end{array}$ & $\begin{array}{l}\text { Masterstudiengang Qualitäts- und Prozessmanagement im } \\
\text { Gesundheitswesen }\end{array}$ & 120 & MSs & $1.453,44$ \\
\hline FH für Gesundheitsberufe in Oberösterreich & Master-Studiengang Management for Health Professionals & 120 & MSc & - \\
\hline \multicolumn{5}{|l|}{ Diplomstudium } \\
\hline UMIT Tirol & Gesundheitswissenschaften & 120 & Mag. & $10.400,-$ \\
\hline \multicolumn{5}{|l|}{ Doktoratsstudien } \\
\hline UMIT Tirol & Doktoratsstudium Gesundheitsinformations-systeme & 180 & Dr. phil. & $21.600,-$ \\
\hline UMIT Tirol & Doktoratsstudium Health Technology Assessment & 180 & Dr. phil. & $21.600,-$ \\
\hline UMIT Tirol & $\begin{array}{l}\text { Doktoratsstudium Management und Ökonomie im Gesund- } \\
\text { heitswesen }\end{array}$ & 180 & Dr. phil. & $21.600,-$ \\
\hline UMIT Tirol & $\begin{array}{l}\text { Doktoratsstudium Sportmedizin, Gesundheitstourismus und } \\
\text { Freizeitwissenschaften }\end{array}$ & 180 & Dr. phil. & $21.600,-$ \\
\hline
\end{tabular}


postgraduale berufsbegleitende Universitätslehrgänge und zwei Doktoratsstudien Public Health angeboten. An der medizinischen Universitäten Wien und der Universität Wien (universitätsübergreifend) sowie an der medizinischen Universität Graz werden Ausbildungen in Public Health angeboten, die einen postgraduellen Erwerb eines Mastertitels Master of Public Health (MPH) ermöglichen. Der geplante Lehrgang Public Health Governance in Vorarlberg, ein zur Medizinischen Universität Graz zu rechnendes Angebot, nimmt eine Sonderstellung ein, da hier ein Abschluss als Master of Science (MSc) angeboten wird. Diese drei Lehrgänge stellen damit das Kernangebot an Public Health Ausbildungen in Österreich dar. Weites werden an Österreichischen Universitäten zwei Doktoratsstudien im Bereich Public Health angeboten, die mit Doctor scientiae medicinae (Dr. scient. med.) bzw. mit Doctor philosophiae (Dr. phil.) abschließen (Universität Wien und Private Universität für Gesundheitswissenschaften, Medizinische Informatik und Technik, abgekürzt UMIT, Tirol).

In Tab. 2 sind die Ausbildungen an österreichischen Universitäten und Fachhochschulen dargestellt, die Public Health neben anderen Themen beinhalten. Es wurden 13 Universitätslehrgänge mit Public Health Inhalten gefunden. Sieben davon sind Lehrgänge für Gesundheitsmanagement bzw. Health Care Management. Die Master Titel, die dafür verliehen werden, sind sehr unterschiedlich, der häufigste davon (bei fünf Lehrgängen) ist der Master of Business Administration (MBA).

In den Lehrgängen können Bachelor- und Mastertitel erworben werden. Bei den sieben Bachelor-Studien an Fachhochschulen, wird sechsmal ein Bachelor of Arts (BA) und einmal Bachelor of Science (BSc) verliehen. Weiters werden neun Master-Studien angeboten, die auf Bachelor-Studien aufbauen. Die Titel, die für diese Abschlüsse verliehen werden, sind entweder Master of Arts (MA) oder Master of Science (MSc).

An der privaten Universität UMIT wird ein Diplomstudium (Gesundheitswissenschaften) angeboten, das mit dem Titel Magister/Magistra der Gesundheitswissenschaften abschließt. Weiters werden an dieser Universität vier Doktoratsstudien (Dr. phil.) angeboten.

Die reinen Public Health Universitätslehrgänge und Public Health Doktoratsstudien werden in Folge eingehender betrachtet.

\section{Universitätslehrgang Master of Public Health der Medizinischen Universität Wien und der Universität Wien}

\section{Lehrgangsbeschreibung}

Dieser Lehrgang vermittelt Kompetenzen und Fähigkeiten auf dem Gebiet der integrativen Prävention und Lebensstilmedizin, um sie zielgruppenorientiertim Sinne der öffentlichen Gesundheit (Public Health) anwenden und evaluieren zu können. Damit verbunden sind der Erwerb von umfassenden Wissensgrundlagen und fachlichen Qualifikationen für Führungsaufgaben und Leitungsfunktionen im Gesundheitswesen mit Schwerpunkt Prävention. Das interdisziplinäre Curriculum des Master-Studienganges in Public Health setzt den Schwerpunkt in der Ausbildung für Lebensstilmedizin mit besonderer Berücksichtigung auf Leistungs-, Ernährungs- und Sozialmedizin, Epidemiologie und Gesundheitsmanagement. Ein Ziel ist der Erwerb von Kompetenzen zur nachhaltigen Umsetzung des Wissens in der Praxis wie im Ambulanzbereich. Hauptziel des Lehrganges ist es, das „Präventionshandwerk“ für die tägliche Praxis im Dienste der öffentlichen Gesundheit zu vermitteln [7].

\section{Zielgruppe und Voraussetzungen}

Der Lehrgang richtet sich an Akademikerinnen und Akademiker, aber auch an Nichtakademikerinnen bzw. Nichtakademiker mit erfolgreicher Studienberechtigung und einschlägiger, mehrjähriger Berufserfahrung. Als postgradueller Lehrgang sind als Zielgruppe Ärztinnen und Ärzte, Zahnärztinnen und Zahnärzte, Gesundheitswissenschafterinnen und Gesundheitswissenschafter (Dr. scient. med.) oder Absolventinnen und Absolventen eines facheinschlägigen naturwissenschaftlichen, wirtschafts-, rechts-, geistes- oder sozialwissenschaftlichen Studiums im In- oder Ausland genannt. Teilnahmevoraussetzung für Personen ohne akademischen Abschluss sind mindestens 5 Jahre Berufserfahrung in fachverwandten Berufen und Matura, zwei Jahre Berufserfahrung oder drei Jahre Führungserfahrung bei Nachweis eines 120 ECTS umfassenden Diplomstudiums.

\section{Dauer und Abschluss}

Der Lehrgang erstreckt sich über vier Präsenzstudiensemester und umfasst 120 ECTS. Er schließt mit dem akademischen Grad Master of Public Health (MPH) ab.

\section{Curriculum}

Beginnend mit einer als „Basisstudium“ bezeichneten Einführungsphase werden in den Basismodulen 1 bis 5 grundlegende Kenntnisse in Biostatistik, Epidemiologie, Environmental Health, Health Service Administration, Social and Behavioral Sciences und Ethik vermittelt. Im Vertiefungsstudium (Module A bis P) geht es zunächst um Grundlagen von Gesundheitswissenschaft, Management und Lifestylemanagement. Drei Module (D, E, H) befassen sich mit Sportmedizin, sportmedizinische Testverfahren und Trainingsberatung sowie mit Ernährung und Stoffwechsel. Weitere Modultitel sind Psychologie, Lebensstilfaktoren, Skilltraining, betriebliche Gesundheitsförderung und spezielle Populationen. Auch wissenschaftliches Arbeiten ist Gegenstand eines Moduls gegen Lehrgangsende. 


\section{Unterrichtsart}

Es handelt sich um ein Präsenzstudium. Zwölf der 120 ECTS können in Form eines Praktikums und weitere neun ECTS über Kongresse und Tagungen erworben werden [7].

\section{Universitätslehrgang Master of Public Health der Medizinischen Universität Graz}

\section{Lehrgangsbeschreibung}

Ziel des Grazer Public-Health-Lehrganges ist es, erfahrenen und motivierten Fachkräften die Möglichkeit zu bieten, sich für eine gehobene Funktion im Gesundheitssystem zu qualifizieren. Im Universitätslehrgang Public Health der Medizinischen Universität Graz erwerben die Absolventinnen und Absolventen eine wissenschaftliche, planerische, gestalterische und gesundheitspolitische Expertise („Wissen und Können“) für die folgenden Aufgabengebiete:

- Epidemiologische Beschreibung, Analyse und Bewertung;

- Vermittlung gesundheitswissenschaftlicher Information;

- Planung, Begleitung und Qualitätsmanagement in der Krankenversorgung (Schwerpunkt Krankenversorgung);

- Planung, Begleitung und Qualitätsmanagement in der Gesundheitsförderung (Schwerpunkt Gesundheitsförderung);

- Organisationsberatung und Mitarbeiterfortbildung [8].

\section{Zielgruppe und Voraussetzungen}

Der Kurs richtet sich an erfahrene Fachpersonen aus Organisationen der Krankenversorgung, Pflege, Rehabilitation und aus der Gesundheitsförderung, aus Bundesministerien, Rechtsabteilungen und Verwaltungen der Bundesländer und Kommunen, aus Sozial- und Privatversicherungen, Kammern und Berufsverbänden, aus der pharmazeutischen Industrie, der Medizintechnikund Gesundheitsgüter- Industrie. Weitere Zielgruppen sind:

- Fachpersonen in einem traditionellen Gesundheitsberuf (Ärztin/Arzt, diplomierte Pflegekraft, Psychotherapeutin/Psychotherapeut)

- Personen mit einem Studienabschluss in einem naturoder ingenieurwissenschaftlichen, einem sozial-, rechts-, wirtschafts- oder geisteswissenschaftlichen Fachbereich, sowie

- Wissenschaftlerinnen, Wissenschaftler und Lehrpersonen, die sich im Bereich von Public Health als der dritten Säule des Gesundheitssystems spezialisieren wollen.

\section{Dauer und Abschluss}

Der Lehrgang erstreckt sich über vier Semester Präsenzstudium und 120 ECTS. Nach Abschluss und Verfassen der Masterthesis wird der Titel Master of Public Health (MPH) verliehen.

\section{Curriculum}

Das Curriculum nennt zwei Schwerpunkte: Management in der Gesundheitsförderung und Management in der Krankenversorgung. Nach einem Einführungsmodul mit 9 ECTS, in dem Konzepte und Methoden der Gesundheitswissenschaften, Sozial-und Selbstkompetenz sowie Informations- und Wissensmanagement behandelt werden, folgen sieben Module mit je 13 ECTS: A: Grundlagen der Gesundheitswissenschaften und von Public Health, B:Grundlagen der Statistik, Demografie und Epidemiologie, C: Organisations- und Managementwissenschaften, D: Grundlagen des Gesundheits-, Sozial- und Krankenversorgungssystems, E: Management in der Gesundheitsförderung, F: Management in der Krankenversorgung und G: Steuerung des Gesundheitssystems. Die Module E-G zählen zur Phase des Vertiefungsstudiums. Die restlichen 20 ECTS werden über die Masterthesis erworben

\section{Unterrichtsmethode}

Der Lehrgang ist ein Präsenzstudium. Maximal 26 ECTS können in einem Public Health Curriuculum einer anderen in- oder ausländischen Universität erworben werden und weitere maximal 5 ECTS über aktive Teilnahme an Fachtagungen oder Kongressen [8].

\section{Universitätslehrgang Public Health Governance Vorarlberg, Schoss Hofen}

Nachdem in Vorarlberg in Kooperation der Erwachsenenbildungseinrichtung des Landes Vorarlberg „Schloss Hofen" mir der Medizinischen Universität Graz zwei Lehrgänge in Public Health veranstaltet und abgeschlossen wurden, deren Curriculum dem des Grazer Lehrgangs entsprach, wurde nach einer Bedarfserhebung bei Sozial- und Gesundheitseinrichtung in Vorarlberg und Österreich ein neues Curriculum mit dem Schwerpunkt Governance entwickelt. Der Lehrgang wurde wegen schleppender Anmeldungen verschoben, ist aber weiterhin im Portfolio von Schloss Hofen und soll zu einem späteren Zeitpunkt gestartet werden. Er wurde daher in die Liste der Masterstudien mit aufgenommen.

\section{Lehrgangsbeschreibung}

Das postgraduale Studium für Public Health Governance setzt seine Schwerpunkte in der Systemsteuerung und -gestaltung des Gesundheitswesens, mit „neuer innovativer Didaktik“ und Ausrichtung auf die Herausforde- 
rungen im regionalen und überregionalen Kontext. Die Besonderheit dieses Lehrgangs ist eine Teilung in einen Studiengang „Akademische/r Fachexperte/Fachexpertin für Public Health Governance" und einen Masterlehrgang. Die ersten vier Präsenzsemester des Masterlehrgangs entsprechen dem Fachexpertenlehrgang [9].

\section{Zielgruppe und Voraussetzungen}

Der Lehrgang richtet sich an interessierte und engagierte Fachkräfte in drei Bereichen: Trägerorganisationen von Gesundheits- und Krankenversorgungsleistungen, Regierung und EU Stellen, Einrichtungen zur Gesundheitsförderung, Einrichtungen der Forschung im Gesundheitsbereich. Aufnahmevoraussetzungen sind ein Abschluss eines international anerkannten, mindestens dreijährigen Hochschulstudiums (Bakkalaureat, Master, Magister/Magistra, Doktorat) plus mindestens 2 Jahre Berufserfahrung im Gesundheitssystem oder eine erfolgreiche Studienberechtigungsprüfung (Matura-Äquivalent) plus mindestens 6-jährige qualifizierte Berufserfahrung in Gesundheitsorganisationen oder in einem gesundheitsnahen Bereich plus erfolgreiche Absolvierung des Universitätslehrgangs Akademische/r Fachexperte/Fachexpertin für Public Health Governance mit überdurchschnittlicher Leistung (Gesamt-Notendurchschnitt $\leq 1,7$ ) plus das Äquivalent eines akademischen Erstabschlusses aus Teilstudien, akademischen Weiterbildungen oder Kursen. Damit wird ausgezeichneten Studierenden, die a priori nicht für den Masterlehrgang qualifiziert sind, der Übertritt ins Masterstudium ermöglicht.

\section{Dauer und Abschluss}

In der Version mit Masterabschluss dauert der Lehrgang 6 Semester - einschließlich der 2 Semester Masterarbeit - (Akad. Fachexperte 4 Semester) und wird berufsbegleitend angeboten. Der Lehrgang findet ausschließlich in Vorarlberg an den Standorten Lochau und Dornbirn statt. Den Absolventinnen und Abvsolventen wird der Titel Master of Science in Public Health Governance (MSc PHG) verliehen, zusätzlich erhalten die Teilnehmerinnen und Teilnehmer auch ein Zeugnis über die Leistungen bzw. erworbenen ECTS aus den einzelnen Modulen.

\section{Curriculum}

Das Curriculum ist in vier Sektionen/Teile und 9 Module aufgeteilt. Sektion A, Wissenschaftliche und methodische Grundlagen der Public Health Wissenschaft, beginnt mit einem Einführungsmodul 1, das die Gruppenbildung, Kennenlernen von Lehrenden und die Lernorganisation (Einführung und Unterweisung der didaktischen Methoden) zum Inhalt hat. Module 2 und 3 sind weitere Bausteine der Sektion A und fokussieren bereits auf Governancemethoden und Prinzipien in Wissenschaft, Politik und Management. Der Governance-
Schwerpunkt setzt sich im Teil B (Patientenorientierte Pflege). Teil C (Systeme der Gesundheitsförderung und Primärprävention) sowie Teil D (Governance in der Entwicklung von Gesundheitssystemen und Koordination im Gesundheitsbereich) fort.

\section{Unterrichtsmethode}

Im ULG Public Health Governance wurde Problem Based Learning (PBL) als vorherrschende didaktische Methode konzipiert. Diese Lernmethode zielt einerseits auf einen nachhaltigen Erwerb nicht nur von Faktenwissen sondern auch von sozialer und persönlicher Kompetenz ab und versucht das Prinzip der Problemorientierung als Vehikel für den Erwerb neuer Fähigkeiten vor dem bisherigen professionellen Hintergrund der Studierenden zu verwenden. Dieser Lernerwerb findet strukturiert in von Tutoren geleiteten Kleingruppen statt. Ergänzt wird PBL durch konventionellen Frontalunterricht und schriftliche Arbeiten [9].

\section{Doktoratsstudium Public der Health Medizinischen Universität Wien}

\section{Lehrgangsbeschreibung}

Das Doktoratsstudium „Doctoral Program of Applied Medical Science“ der Medizinischen Universität Wien kann den Bereich Public Health als sogenanntes Thematisches Programm als Schwerpunkt beinhalten und wird damit hier in die Reihe der reinen Public Health Studiengänge aufgenommen. $\mathrm{Zu}$ den Zielen dieses Doktoratsstudiums gehört die Entwicklung von Kompetenzen für selbständiges wissenschaftliches Arbeiten durch den Erwerb von kritischen, analytischen und argumentativen Fähigkeiten. Es wird eine Qualifikation zur Ausübung von Wissenschaft als Beruf angestrebt. Absolventinnen und Absolventen sollen unter anderem die Fähigkeit erwerben, Forschungsprojekte zu planen, zu entwerfen und umzusetzen, substanzielle Forschungsergebnisse zu produzieren, internationale Publikationsstandards zu beherrschen und schließlich die Befähigung besitzen, sowohl in der wissenschaftlichen als auch der Gesellschaft im Allgemeinen erfolgreich zu kommunizieren [10].

\section{Zielgruppe und Voraussetzungen}

Die Zulassung zu diesem Studium erhalten Absolventinnen und Absolventen eines Medizin-oder Zahnmedizinstudiums, naturwissenschaftlicher Studien oder relevanter Diplomstudien sowie Absolventinnen und Absolventen eines Masterlehrgangs, sofern das Rektorat darin eine entsprechende Äquivalenz zu den erstgenannten Studien sieht. Angesprochen werden vor allem Interessierte mit einem stark klinisch-wissenschaftlichen Schwerpunkt, die für die Entwicklung ihrer Doktorarbeit eine Umgebung suchen, in der sowohl Nachwuchsfor- 
scherinnen und -forscher als auch Praktikerinnen und Praktiker optimale Bedingungen vorfinden, in denen Forschungsprojekte durchgeführt und weiterentwickelt werden können.

\section{Dauer und Abschluss}

Die Mindeststudienzeit beträgt 6 Semester mit gesamt 180 ECTS-Punkten. Absolventinnen und Absolventen erwerben den Grad eines Doctor scientiae medicinae (Dr. scient. med.).

\section{Curriculum}

Innerhalb des Themenprogramms Public Health müssen aus dem Kursangebot 4 Semesterstunden Basisseminare, 12 Semesterstunden Journal Clubs (siehe unten), 8 Semesterwochenstunden Thesis-Seminare und 6 Semesterstunden Propädeutikum nachgewiesen werden. Spätestens bis zum Ende des ersten Semesters muss eine Betreuerin oder ein Betreuer (Supvervisor) für die Dissertation und ein Thema festgelegt werden. Das Thema der Dissertation soll nach Möglichkeit mit einem peerreviewed Forschungsprojekt assoziiert sein.

\section{Unterrichtsmethode}

An Unterrichtsveranstaltungen werden im Einzelnen folgende Kurstypen beschrieben: Vorlesungen und Seminare dienen der Vermittlung von Basiswissen und -methoden, die professionelle Anwendung wird in Praktika geübt. Journal Clubs dienen dem Erlernen von kritischer Auseinandersetzung mit Publikationen und Studien. Einige Kurse sind Pflichtveranstaltungen für alle Studierenden, schließlich gibt es noch Thesis-Seminare zur Erarbeitung spezieller Aspekte der jeweiligen Dissertationsthemen [10].

\section{Doktoratsstudium Public Health der UMIT Hall/Tirol}

\section{Lehrgangsbeschreibung}

Das Doktoratsstudium Public Health der Privatuniversität UMIT in Hall soll die Befähigung vermitteln selbstständig wissenschaftliche Fragenstelllungen aus dem Gebiet Public Health zu entwickeln und Forschungsprojekte zu konzipieren und durchzuführen. Neben dem Erlernen und der Vertiefung von entsprechenden Kenntnissen über moderne qualitative und quantitative Methoden wird eine Einbindung der Studierenden in die bestehende Forschungslandschaft und in laufende Spitzenforschungsprojekte des nach Angaben der UMIT national und international exzellent vernetzten Departements für Public Health und Health Technology Assessment in Aussicht gestellt [11].

\section{Zielgruppe und Voraussetzungen}

Zugelassen sind Absolventinnen und Absolventen mit einem Bachelor-, Master-, Magister- oder Diplomabschluss einer anerkannten Universität oder eines mindestens 10-semestrigen Studiums aus Fachrichtungen Public Health, Gesundheitswissenschaften, Medizin, Zahnmedizin, Epidemiologie, Statistik, Pharmazie, Biologie, Pflegewissenschaft, Psychologie, Informatik, Health Technology Assessment, Gesundheitsmanagement, Politikwissenschaften, Rechtswissenschaften, Wirtschaftswissenschaft, Kommunikationswissenschaften sowie sozialwissenschaftliche, philosophische und naturwissenschaftliche Studien, sofern hinsichtlich dieser Studien im Rahmen von generellen oder individuellen Schwerpunktsetzungen wesentliche Bezüge zum Gesundheitswesen nachgewiesen werden können. Damit wird der Personenkreis von Absolventinnen und Absolventen einer Vielzahl von Studien aus dem Umfeld der Multidisziplin Public Health angesprochen. In der Studienbeschreibung findet sich auch der Hinweis, dass von den zirka 200 Absolventinnen und Absolventen eines Doktorats in Public Health im deutschsprachigen Raum die Mehrzahl in Führungsfunktionen im Gesundheitsbereich und zum kleineren Teil in der Forschung tätig ist.

\section{Dauer und Abschluss}

Das Studium erfordert den Erwerb von 180 ECTS Punkten und den Nachweis einer Dissertationsvereinbarung und einer Betreuungsbestätigung. Es ist mit einer Studiendauer von sechs Semestern geblockt angelegt. Absolventinnen und Absolventen erhalten den akademischen Grad eines Doctor philosophiae (Dr. phil.).

\section{Curriculum}

Bei Vorliegen der Dissertationsvereinbarung und einem festgelegten Thema kann mit der Betreuerin oder dem Betreuer und in Absprache mit der Studienleitung eine individuelle Zusammensetzung von Vorlesungen und Veranstaltungen für den Erwerb der ECTS erstellt werden. Dazu findet sich ein umfassendes Angebot aus den an der UMIT angebotenen Bachelor-, Master- und Doktoratsstudien wie z. B. Gesundheitsinformationssysteme, Health Technology Assessment, Management und Ökonomie in Gesundheitswesen, Pflegewissenschaft etc. Weiters sind im Rahmen des Doktoratsstudiums neben der wissenschaftlichen Forschungsleistung im Durchschnitt 10 ECTS pro Semester über Veranstaltungen (z. B. Methodenlehre, wissenschaftliches Schreiben, Vertiefungsfächer, Privatissima etc.) zu absolvieren.

\section{Unterrichtsmethode}

Die Ausbildung ist ein Präsenzstudium [11]. 


\section{Public Health in medizinischen Curricula}

Die Tatsache, dass für angehende Ärztinnen und Ärzte nicht nur medizinisch-klinisches Wissen und Fertigkeiten nötig ist, sondern dass Ärztinnen und Ärzte maßgebliche Player im Gesundheitssystem sind, in dem sie sich zurecht finden müssen, und zusätzlich Ärztinnen und Ärzten eine immer größer werdende Rolle nicht nur in der kurativen Medizin sondern auch in der präventiven Medizin und in der Gesundheitsförderung zukommt, hat zur Etablierung von Public Health Inhalten in den Ausbildungscurricula den Medizinischen Universitäten geführt. War es in früheren Curricula, bei denen im Fächerkanon die einzelnen medizinischen Disziplinen nacheinander absolviert wurden, insbesondere das Fach Sozialmedizin, in dem Public Health relevante Inhalte gelehrt und in Form des Teilrigorosums Sozialmedizin geprüft wurde, sind es in den modernen Spiralcurricula Public Health Inhalte, meist an verschiedenen Stellen des Gesamtcurriculums, in denen der Zusammenhang von Public Health mit klinischen Ausbildungsinhalten bzw. die Relevanz von Public Health als Vorbereitung auf den ärztlichen Beruf klar vermittelt wird. Public Health Inhalte und das Ausmaß von Public Health in den medizinischen Curricula unterscheiden sich jedoch an den vier Österreichischen Medizinischen Universitäten und werden in der Folge dargestellt.

\section{Medizinische Universität Wien}

Im Medizinstudium an der Medizinischen Universität Wien wird Public Health vor allem an zwei Stellen des Pflichtcurriculums gelehrt: Bereits im zweiten Semester werden im Block 6 mit dem Titel „Der Mensch in Umwelt, Familie und Gesellschaft“ wesentliche Grundlagen von Public Health vermittelt. Hier geht es um Gesundheitsdefinitionen und Gesundheitsmodelle, umweltbezogene Einflüsse auf die Gesundheit, Grundlagen der Epidemiologie, Screening, soziale Gesundheitsdeterminanten, Prävention sowie um kulturanthropologische und ethnologische Grundlagen der Medizin [12]. Dieser Block beinhaltet insgesamt 63 akademische Stunden angeleiteten Unterricht. Im 7. Semester werden diese Public Health Themen in einem mit dem Titel „Public Health. Von den Gesundheitsbedürfnissen der Gesellschaft zu klinischen Implikationen“ vertieft. Dieser Block stellt mit 100 akademischen Stunden einen der umfangreichsten Blöcke des gesamten Medizinstudiums dar. Der Block beinhaltet Themen wie Aufbau des Gesundheitssystems, Ebenen der Gesundheitsversorgung, Integrierte Versorgung, Patientenorientierte Versorgung, Grundlagen der Gesundheitsökonomie und der Versicherungsmedizin, Evidence Based Medicine und Health Technology Assessment sowie Qualitätssicherung und Fehlermanagement im Gesundheitssystem. Weitere Kapitel in diesem Block beschäftigen sich mit Medizinrecht, Ethik in der Medizin und im Gesundheitswesen, Präventivmedizin inklusive Arbeitsmedizin, Umweltmedizin, Reisemedizin und Impfungen und Rehabilitation, sowie den gesundheitlichen Herausforderungen für das Gesundheitssystem durch die demographische Entwicklung im Kapitel Geriatrie [13]. Das Lehrausmaß Public Health im Wiener Medizincurriculum entspricht somit 165 akademischen Stunden bzw. 11 Semesterwochenstunden.

\section{Medizinische Universität Graz}

An der Medizinischen Universität Graz werden Public Health Relevante Inhalte in erster Linie im 5. Studiensemester im Modul 15 mit dem Titel „Gesundheit und Gesellschaft" gelehrt. Dieser Modul beinhaltet die Interaktion von Mensch und Gesellschaft, Grundlagen der öffentlichen Gesundheit und des Gesundheitswesens und Grundzüge der Epidemiologie und der Präventivmedizin. Das Lehrausmaß Public Health im Grazer Medizincurriculum umfasst 8 Semesterwochenstunden bzw. 8 ECTS [14].

\section{Medizinische Universität Innsbruck}

An der Medizinischen Universität Innsbruck wird im 7. Semester das Modul 2.31 mit dem Titel „Mensch in Familie, Gesellschaft und Umwelt" mit 4,5 Semesterstunden gelehrt. Ein weiteres Modul im 8. Semester (Modul 3.06) beschäftigt sich in 1 Semesterstunde mit „Biostatistik und Evidence Based Medicine“ [15]. Das Lehrausmaß Public Health im Innsbrucker Medizincurriculum umfasst somit 5,5 Semesterwochenstunden.

\section{Paracelsus Medizinische Privatuniversität Salzburg}

An der Paracelsus Medizinischen Privatuniversität Salzburg werden gemäß Studienplan Public Health relevante Inhalte an verschiedenen Stellen des Curriculums gelehrt. Im ersten Jahr werden Grundlagen der Medizinischen Ethik vermittelt, im dritten Jahr wird Allgemeinund Familienmedizin genauer betrachtet, im vierten Jahr werden Inhalte der klinischen Epidemiologie und der medizinischen Ökonomie gelehrt. Schließlich stehen im fünften Jahr die Inhalte Präventivmedizin, rechtliche Aspekte der Medizin und Public Health am Lehrplan [16].

\section{Diskussion}

In diesem Artikel wurden Public Health relevante Studien und Lehrgänge an Österreichischen Universitäten und Hochschulen recherchiert. Es konnten 3 reine Public Health Lehrgänge und 2 reine Public Health Doktoratsstudien identifiziert werden. Zusätzlich konnten 34 Studiengänge identifiziert werden, in denen Teilbereiche von Public Health abgedeckt sind. Die Inhalte dieser Studiengänge sind sehr unterschiedlich, ebenso wie das vorausgesetzte und das zu erreichende Ausbildungsniveau. Ein reines Public Health Diplomstudium bzw. ein reines Public Health Studium, das entsprechend den Zyklen des 
Bologna Prozesses Bachelor- Master- und PhD- Qualifikationen aufbaut, gibt es in Österreich zur Zeit nicht.

Die meisten der dargestellten Studien und Lehrgängen mit Public Health Inhalt wurden nach der Jahrhundertwende initiiert und sind entsprechend jung. Die Tiefe des wissenschaftlichen Background und das Ausmaß von Public Health Erfahrung der lehrenden Personen ist in vielen Kursen ungewiss. In dieser Darstellung der Lehrgänge und Studien können keine Schlüsse über Relevanz und Qualität der Public Health Inhalte gezogen werden, aber das ist auch nicht der Focus dieser Arbeit. Generell ist die online verfügbare Information zu den Lehrgängen, deren Schwerpunkte und generelle Ausrichtung durchwegs leicht zu finden. Dies erlaubt den Interessierten einen einfachen Vergleich der Studiengänge.

Limitierend $\mathrm{zu}$ dieser Recherche muss angemerkt werden, dass lediglich Ausbildungen angeführt wurden, die an österreichischen Universitäten zum Zeitpunkt der Manuskripterstellung (Herbst 2013) angeboten werden. Zur Darstellung gelangten hier bis auf wenige Ausnahmen, die aufgrund der deutlichen Public Health Relevanz aufgenommen wurden, außerdem lediglich Studiengänge, die „Public Health“, „Health“, oder „Gesundheits-“ in ihrem Titel enthielten. Es kann somit sein, dass es Studiengänge gibt, die Public Health relevante Aspekte anbieten und nicht in dieser Auflistung aufscheinen. Dazu zählen Studien aller Public Health assoziierter Disziplinen, wie beispielsweise Soziologie, Psychologie, Ernährungswissenschaften, Sportwissenschaften, Ökonomie, Politikwissenschaften, Pflegewissenschaften oder Ausbildungen für verschiedene Health Professionals. Um diese Limitierung etwas abzuschwächen, wurde in diesem Artikel auf die Public Health Inhalte der bestehenden Medizincurricula in Österreich verwiesen.

Es muss Gegenstand von zukünftigen Diskussionen und darauffolgender weitsichtiger Planung sein, inwieweit die Gestaltung neuer, einheitlicher oder aber spezialisierter Lehrgänge den steigenden Bedarf an Personal im Bereich Public Health decken kann. Inhomogenität oder Variantenreichtum beim Schwerpunkt der Lehrgänge (Prävention/Gesundheitssysteme/Management/ Governance etc) können durchaus ein Anreiz für Professionals unterschiedlicher Provenienz sein, eine Public Health (Zusatz-)Ausbildung anzustreben. Gleichzeitig braucht es aber auch einen gemeinsamen Wissensstand der grundlegenden Kenntnisse bei den Public Health Professionals. Eine Akkreditierung des Ausbildungslehrgangs z. B. bei der European Agency for Accreditation in Public Health Education (APHEA) setzt einen solchen Standard voraus und deren Vorhandensein sollte bei der Auswahl des Lehrgangs eine Grundvoraussetzung sein[17]. Als Kerngebiete nennen die ASPHEA-Richtlinien Biostatistik, Epidemiologie, Gesundheitsdeterminanten, Gesundheitspolitik, Gesundheitsökonomie, Prävention und Gesundheitsförderung sowie fachübergreifendes Grundlagenwissen.

Um ein in allen Ebenen des Gesundheitswesens ausreichend vorhandenes Public Health Verständnis zu erzeugen, muss eine kritische Masse an Expertinnen und Experten mobilisiert, ausgebildet und im Gesundheitswesen, im Sinne von Health in all Policies auch in anderen Verantwortungsräumen, platziert werden, wo sie mit anderen Public Health Professionals ihr Wissen teilen und in einem gemeinsamen Verstehen über die Herausforderungen des Gesundheitssystems handeln können. Wird diese kritische Masse erreicht, entsteht also ein bestimmtes Maß an „Public Health Awareness“, steigt auch die Nachfrage nach entsprechender Aus- und Weiterbildung und damit die Qualität und Quantität des Angebots. Von dieser Triggerschwelle dürfte Österreich jedoch noch weit entfernt sein. Dies mag mit der verzögerten Präsenz vor allen der „New Public Health" Themen wie gesellschaftlicher Einfluss auf Gesundheit und Krankheit, sozial bedingte ungleiche Gesundheitschancen, Verständnis von Gesundheit als Resultat einer Balance zwischen Belastungen und Ressourcen [18] zu tun haben, aber auch mit historisch verwurzelten Medizinzentriertheit des österreichischen Gesundheitssystems.

Zweifellos ist mit der Formulierung und Verankerung der Rahmengesundheitsziele in Österreich [19], an denen im Moment gearbeitet wird, ein wichtiger Schritt in Richtung Public Health gesetzt worden[20]. Die Tatsache, dass nur eines von zehn Zielen die Gesundheitsversorgung per se zum Thema hat zeigt, dass die Prinzipien von Health in all Policies und das Verständnis für eine multidimensionale und multisektorale Verantwortung für eine gesunde österreichische Bevölkerung implementiert werden. Um die Herausforderungen $\mathrm{zu}$ meistern, die die Umsetzung dieser Ziele mit sich bringen wird, braucht Österreich eine entsprechende Public Health Workforce, also mehr Public Health Expertise in allen Verantwortungs- und Entscheidungsräumen.

\section{Interessenkonflikt}

Die Autoren geben an, dass kein Interessenskonflikt zu den im Artikel präsentierten Ergebnissen besteht.

\section{Open Access}

Dieser Artikel unterliegt den Bedingungen der Creative Commons Attribution Noncommercial License. Dadurch sind die nichtkommerzielle Nutzung, Verteilung und Reproduktion erlaubt, sofern der/die Originalautor/en und die Quelle angegeben sind.

\section{Literatur}

1. Beaglehole R, Bonita R, Horton R, et al. Public health in the new era: improving health through collective action. Lancet. 2004;363:2084-6.

2. Noack H. Public Health in Europa. In: Schwartz FW, editor. Das Public Health Buch: Gesundheit und Gesundheitswesen. 2. Aufl. Jena: Urban und Fischer; 2003. (31.1:757).

3. Medizinische Universität Wien. (2013) http://www.meduniwien.ac.at/homepage/content/allgemeine-informationen/studien-und-weiterbildungsangebot/. Zugegriffen: 12. Sept. 2013. 
4. Medizinische Universität Graz. (2013) http://www.medunigraz.at/ps. Zugegriffen: 12. Sept. 2013.

5. Bundesministerium für Wissenschaft und Forschung. (2013) http://www.studienwahl.at/. Zugegriffen: 12. Sept. 2013.

6. Bundesministerium für Wissenschaft und Forschung (2013). http://www.bmwf.gv.at/Startseite/Hochschulen. Zugegriffen: 12. Sept. 2013.

7. Medizinische Universität Wien (2013). http://www.meduniwien.ac.at/orgs/index.php?id=360. Zugegriffen: 12 . Sept. 2013.

8. Medizinische Universität Graz. (2013) http://www.medunigraz.at/ph. Zugegriffen: 12. Sept. 2013.

9. Medizinische Universität Graz. (2013) www.medunigraz. at/mphg. Zugegriffen: 12. Sept. 2013.

10. Medizinische Universität Wien. (2013) http://www.meduniwien.ac.at/studienabteilung/content/studium-lehre/ studienangebot $/ \mathrm{n} 790 /$ ?Fsize $=0$. Zugegriffen: 12. Sept. 2013.

11. UMIT - Private Universität für Gesundheitswissenschaften, Medizinische Informatik und Technik. (2013) http:// www.umit.at/page.cfm?vpath=studien/doktorat_studien/ public-health/fakten-zum-studium. Zugegriffen: 12. Sept. 2013.

12. Wittmann K, Schoberberger R, editors. MCW Block 6. Der Mensch in Umwelt, Familie und Gesellschaft. 9th ed. Wien: Facultas Verlags- und Buchhandels AG; 2011.
13. Dorner TE, editor. Public Health. Von den Gesundheitsbedürfnissen der Gesellschaft zu klinischen Implikationen. MCW Block 22/23. Wien: Facultas Verlags- und Buchhandels AG; 2013.

14. Medizinische Universität Graz (2013). Studienplan für das Diplomstudiem Humanmedizin. http://www.meduni-graz.at/images/content/file/studium/humanmedizin/ pdf/studienplan_v11_01102013.pdf. Zugegriffen: 12. Sept. 2013.

15. Medizinische Universität Innsbruck. (2012). Mitteilungsblatt der Medizinischen Universität Innsbruck 164. Änderung des Studienplans der Humanmedizin der Medizinischen Universität Innsbruck. https://www.i-med.ac.at/ mitteilungsblatt/2011/40.pdf. Zugegriffen: 12. Sept. 2013.

16. Paracelsus Medizinische Privatuniversität (2013). http:// www.pmu.ac.at/studium/humanmedizin/studienaufbau. html. Zugegriffen: 12. Sept. 2013.

17. Otok R, Levin I, Sitko S, Flahault A. European accreditation of public health education. Public Health Rev. 2011;33:30-8.

18. Rosenbrock R. Was ist New Public Health? Bundesgesundheitsbl - Gesundheitsforsch - Gesundheitsschutz. 2001;44:753-62.

19. Bundesministerium für Gesundheit. (2013) http://www. gesundheitsziele-oesterreich.at/. Zugegriffen: 12. Sept. 2013.

20. Noack H. Leitprinzipien der Gesundheitsbildung für eine gesündere Gesellschaft. Soziale Sicherheit. 2013;9:424-32. 